\title{
Reduced-sized monolayer carbon nitride nanosheets for highly improved photoresponse for cell imaging and photocatalysis
}

\author{
Qinghua Liang ${ }^{1,2,3}$, Zhi Li $^{2}$, Yu Bai ${ }^{3}$, Zheng-Hong Huang ${ }^{3 *}$, Feiyu Kang ${ }^{1,3}$ and Quan-Hong Yang ${ }^{14^{*}}$
}

\begin{abstract}
Two-dimensional graphitic carbon nitride $\left(\mathrm{g}-\mathrm{C}_{3} \mathrm{~N}_{4}\right)$ nanosheets $(\mathrm{GCNNs})$ have been considered as an attractive metal-free semiconductor because of their superior catalytic, optical, and electronic properties. However, it is still challenging to prepare monolayer GCNNs with a reduced lateral size in nanoscale. Herein, a highly efficient ultrasonic technique was used to prepare nanosized monolayer graphitic carbon nitride nanosheets (NMGCNs) with a thickness of around $0.6 \mathrm{~nm}$ and an average lateral size of about $55 \mathrm{~nm}$. With a reduced lateral size yet monolayer thickness, NMGCNs show unique photo-responsive properties as compared to both large-sized GCNNs and GCN quantum dots. A dispersion of NMGCNs in water has good stability and exhibits strong blue fluorescence with a high quantum yield of $32 \%$, showing good biocompatibility for cell imaging. Besides, compared to the multilayer GCNNs, NMGCNs show a highly improved photocatalysis under visible light irradiation. Overall, NMGCNs, characterized with monolayer and nanosized lateral dimension, fill the gap between large size (very high aspect ratio) and quantum dot-like counterparts, and show great potential applications as sensors, photo-related and electronic devices.
\end{abstract}

Keywords: graphitic carbon nitrides, monolayer nanosheets, photocatalysis, fluorescence, cell imaging

\section{INTRODUCTION}

Due to the fascinating physicochemical and electronic properties arising from the small dimensions and quantum confinement effects (QCE) [1-3], ultrathin nanosheets have been the focus of substantial research in the light of fundamental studies and their broad applications in various fields, including optoelectronic devices [4], energy conversion and storage [5], sensors [6-9], biomedicine [10], and catalysis [11-15]. In particular, polymeric graphitic carbon nitride nanosheets (GCNNs), an n-type semiconductor, have been receiving considerable attention due to their many intriguing advantages such as the fact that they contain no metal, and nontoxicity, easy availability, sensitive photo-response, excellent chemical and thermal stability, and unique electronic structures [16-19].

Compared with bulk graphitic carbon nitride (GCN), the remarkable optical and catalytic properties of the GCNNs are mainly associated with the QCE and their larger specific surface area (SSA) $[18,20]$. On one hand, the wider energy bandgap arising from the QCE accompanied by the different photo-physical behavior of photogenerated electron-hole pairs is very helpful for improving their redox performance. On the other hand, the larger SSA of the ultrathin GCNNs provides abundant exposed surfaces, numerous active sites, and a short transfer length for increasing the separation efficiency of interface photoexcited charge carriers, thus showing a much improved photocatalytic performance. In addition, it has been demonstrated that reduced-sized GCNNs and GCN quantum dots with much smaller lateral dimension can be used for bio-imaging due to their distinct fluorescence with low photo-bleaching and low cytotoxicity [21]. However, multilayer GCNNs reported so far have a low fluorescence quantum yield (less than 20\%) $[8,9,22]$, and GCN quantum dots show ignorable photocatalytic activity under visible light irradiation [23]. It is believed that nanosized monolayer graphitic carbon nitride nanosheets (NMGCNs)

\footnotetext{
${ }^{1}$ Shenzhen Key Laboratory for Graphene-based Materials, Graduate School at Shenzhen, Tsinghua University, Shenzhen 518055, China

${ }^{2}$ Technical Institute of Physics and Chemistry, Chinese Academy of Sciences (CAS), Beijing 100190, China

${ }^{3}$ Key Laboratory of Advanced Materials (Ministry of Education), School of Materials Science and Engineering, Tsinghua University, Beijing 100084, China

${ }^{4}$ School of Chemical Engineering and Technology, Tianjin University, Tianjin 300072, China

* Corresponding authors (emails: yang.quanhong@sz.tsinghua.edu.cn or qhyangcn@tju.edu.cn (Yang QH); zhhuang@tsinghua.edu.cn (Huang ZH))
} 
would be highly photo-responsive for both photocatalysis and fluorescence.

Thus far, GCNNs prepared with various techniques, such as mechanical milling $[24,25]$, chemical oxidation thermal or etching $[20,26,27]$, physical and liquid exfoliation [22,28,29], and electrochemical synthesis [30] usually show wide distributions in both the number of layers and lateral size. In particular, due to many advantages of easy operation, low-cost, environmental benignity, and no reason for interfacial defects, liquid-phase exfoliation, usually in conjunction with ultrasonic treatment has been intensively explored for producing ultrathin GCNNs by delaminating bulk GCN $[28,31,32]$. However, the obtained GCNNs usually have a thickness larger than $2 \mathrm{~nm}$ (> 5 layers) $[30,33]$. It is still a challenge to prepare atom-thin GCNN with a reduced lateral size $(<100 \mathrm{~nm})$ although several research groups have recently reported the preparation of monolayer GCNN that has a lateral dimension larger than $1.0 \mu \mathrm{m}[13,34]$. Also, the liquid-phase exfoliation technique is still limited due to its low mass yield (less than $10 \%$ ) and long time required. Previous results also suggest that protonation before sonication not only significantly enhances the product yield of GCNN but also provides a GCNN with better dispersibility in water at a high concentration since the bridged nitrogen of GCN serves as a unique adsorption site that facilitates water intercalation for exfoliation [7]. Furthermore, it has been reported that probe sonication treatment by a high-pressure cell cracker is efficient in breaking a large nanosheet into smaller ones without changing the chemical structure [35]. Here we present a preparation technique for NMGCNs that have an average lateral size of $55 \mathrm{~nm}$ by probe sonication combined with bath sonication using protonated GCNN as the starting material. These NMGCNs show uniqueness to their counterparts, large size GCNNs and GCN quantum dots, and simultaneously possess highly photo-response for cell imaging and photocatalysis.

\section{RESULTS AND DISCUSSION}

The preparation process of the NMGCNs is illustrated in Fig. 1. This synthesis strategy combines bath and probe sonications to exfoliate and tailor multilayer GCNNs into NMGCNs. GCNNs with an average thickness of about 20 $\mathrm{nm}$ were first prepared by the modified thermal poly-condensation of urea in air (Fig. S1, Supplementary information) [36]. It is noted that the high SSA of the GCNNs ( 102 $\left.\mathrm{m}^{2} \mathrm{~g}^{-1}\right)$ is conducive to ultrasonic exfoliation since there is a larger contact area with water (Fig. S2). Multilayer GCNNs were then produced by bath sonication in water using proton-functionalized GCNNs as the starting material. Finally, NMGCNs were obtained with a yield of $\sim 30 \%$ after a strong physical cleavage of the multilayer GCNNs by probe sonication in a conical tube (see Supplementary information). It should be noted that the probe sonication yields a

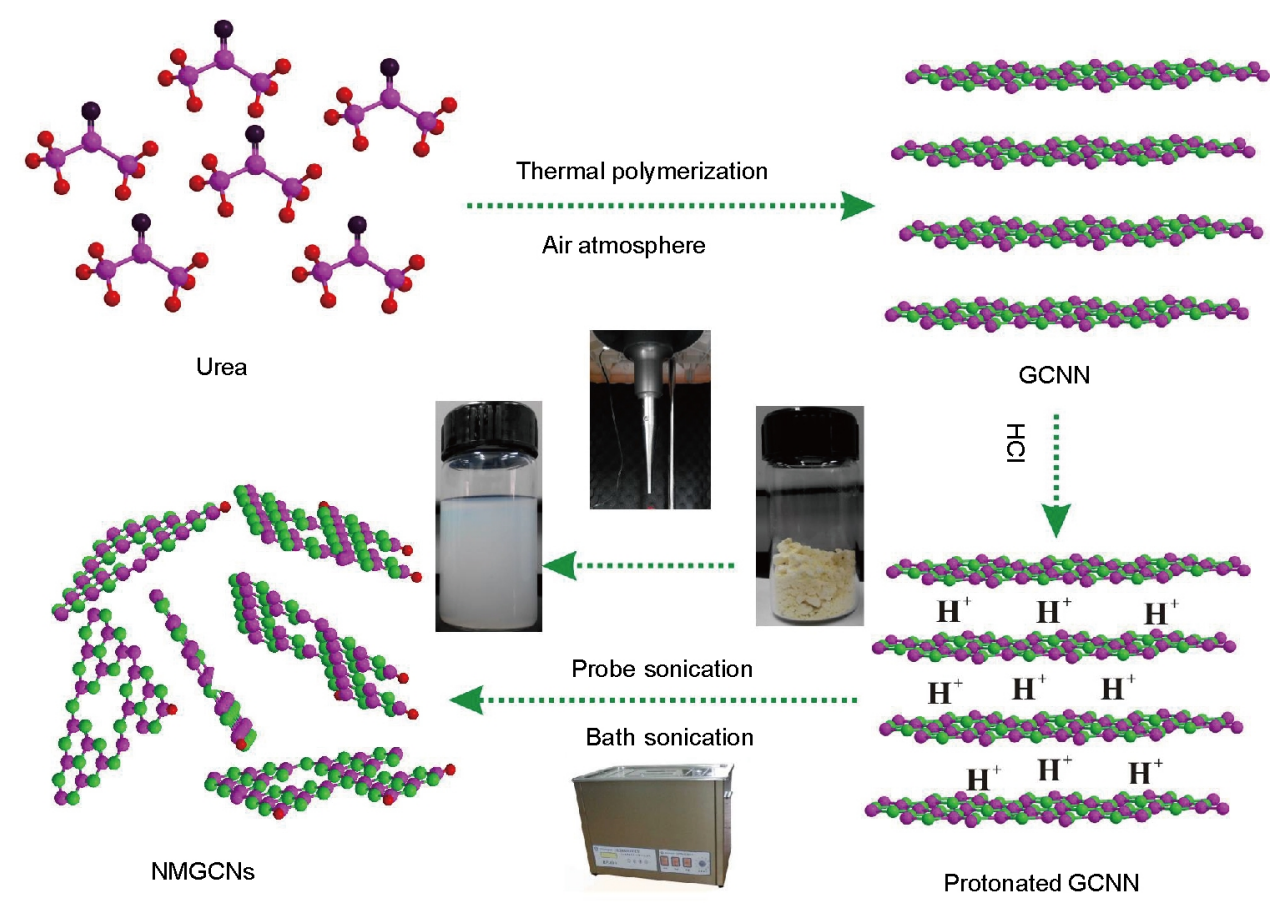

Figure 1 Illustration for the preparation of NMGCNs. 
reduced lateral size for NMGCNs compared with their GCNN counterpart and dot-like NMGCNs with an average size of $3 \mathrm{~nm}$ can be also produced by increasing the sonication time up to $9 \mathrm{~h}$ (Fig. S3).

Atomic force microscopy (AFM) was first carried out to observe the morphology of the NMGCNs. A typical lowmagnification AFM image (Fig. 2a) clearly reveals that the NMGCNs are irregularly shaped nanosheets. Statistical analysis by the ImageJ software reveals that the average lateral size of the NMGCNs is about $55 \mathrm{~nm}$ (Fig. 2b). As demonstrated in Fig. 2c, AFM images of the NMGCNs also reveal freestanding nanosheets with a flat surface. Their average thickness of about $0.60 \mathrm{~nm}$, determined from the fitted height profile measured in AFM topography, verifies their monolayer structure considering the theoretical interlayer distance of GCN is around $0.35 \mathrm{~nm}$ (Fig. 2d). Transmission electron microscopy (TEM) was further conducted to examine the dimensions of the NMGCNs. The low contrast TEM image shown in Fig. 2e confirms their ultrathin nature. The corresponding selected area electron diffraction (SAED) patterns show obscure rings, demonstrating the disordered structures in the NMGCN. High-resolution
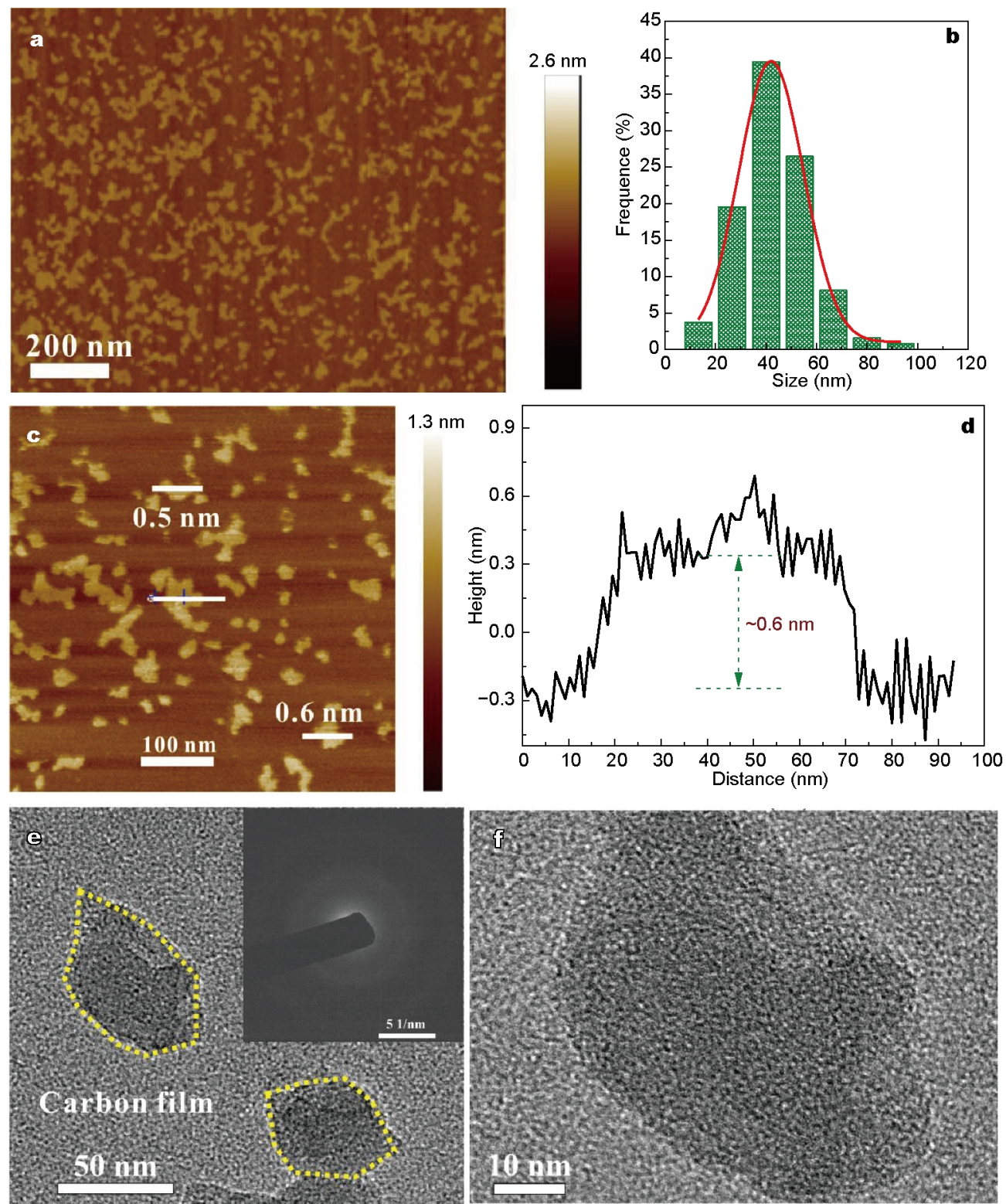

Figure 2 (a) Low-magnification AFM image, (b) the corresponding lateral size distribution, (c) high-magnification AFM image, (d) the corresponding height profile, and (e) TEM and (f) HRTEM images of the NMGCNs. The inset in (e) is the corresponding SAED pattern of the NMGCNs. 
TEM image (HRTEM) also reveals that there is no long-range ordering and continuous lattice fringe in the NMGCNs (Fig. 2f), which is in agreement with the SAED and X-ray diffraction (XRD) results. Both AFM and TEM results confirm the small lateral size and monolayer structure of the NMGCNs.

The layered structure of the NMGCNs was also studied by XRD. Compared with GCNNs (Fig. 3a), the (002) peak at about $27.5^{\circ}$ is much weaker and broader, and the (100) peak at $13.2^{\circ}$ has disappeared, indicating that the stacking of the conjugated aromatic systems and the in-plane repeat units of GCN are obviously disrupted after the exfoliation $[20,26,37]$. A Fourier transform infrared (FTIR) spectrum measurement was conducted to verify the chemical structure of the NMGCNs. As shown in Fig. 3b, the FTIR spectra of both GCNN and NMGCN show a sharp peak at $808 \mathrm{~cm}^{-1}$, five strong peaks at 1240,1320, 1400, 1460, and $1550 \mathrm{~cm}^{-1}$, and a board peak ranging from 3000 to 3500 $\mathrm{cm}^{-1}$, respectively corresponding to the typical breathing vibration of the $s$-triazine ring, the characteristic stretching modes of $\mathrm{CN}$ heterocycles, and stretching of $\mathrm{N}-\mathrm{H}$ and O-H bonds $[26,29,38]$. The FTIR result indicates that the
NMGCNs retain the basic structure of the GCN. Notably, all the FTIR peaks of NMGCNs are much sharper than those of GCNNs, suggesting a larger stacking distance and shorter-range order of the in-plane structure in the NMGCNs. This is in agreement with the above XRD results. Survey X-ray photoelectron spectroscopy (XPS) confirms the presence of $\mathrm{C}, \mathrm{N}$, and $\mathrm{O}$ in the NMGCNs with a $\mathrm{C} / \mathrm{N}$ molar ratio of about 0.76 (Fig. S4), indicating that the pristine backbone of GCN is retained in the NMGCNs. The oxygen is ascribed to a small amount of water adsorbed on the surface of the NMGCNs (Fig. S5). The high resolution $\mathrm{C}$ 1s spectrum shown in Fig. $3 \mathrm{c}$ reveals the existence of two types of $\mathrm{C}$ species including the $\mathrm{C}-\mathrm{C}$ at $284.9 \mathrm{eV}$ and the $\mathrm{C}=\mathrm{N}$ at $288.4 \mathrm{eV}$ [39-41]. The fitted high resolution $\mathrm{N}$ 1s spectrum shows the presence of four types of $\mathrm{N}$ species, containing $\mathrm{C}=\mathrm{N}-\mathrm{C}$ at $398.6 \mathrm{eV}, \mathrm{N}-(\mathrm{C})_{3}$ at $399.5 \mathrm{eV}, \mathrm{C}-\mathrm{N}-\mathrm{H}$ at $400.8 \mathrm{eV}$, and the positive charge of heptazine at $404.8 \mathrm{eV}$ (Fig. 3d) $[39,42,43]$. Both C 1s and $\mathrm{N}$ 1s spectra firmly demonstrate the presence of basic tri-s-triazine units in NMGCNs, in agreement with the FTIR results. The retention of the basic heptazine structure is very important for keeping the unique photo-responsive
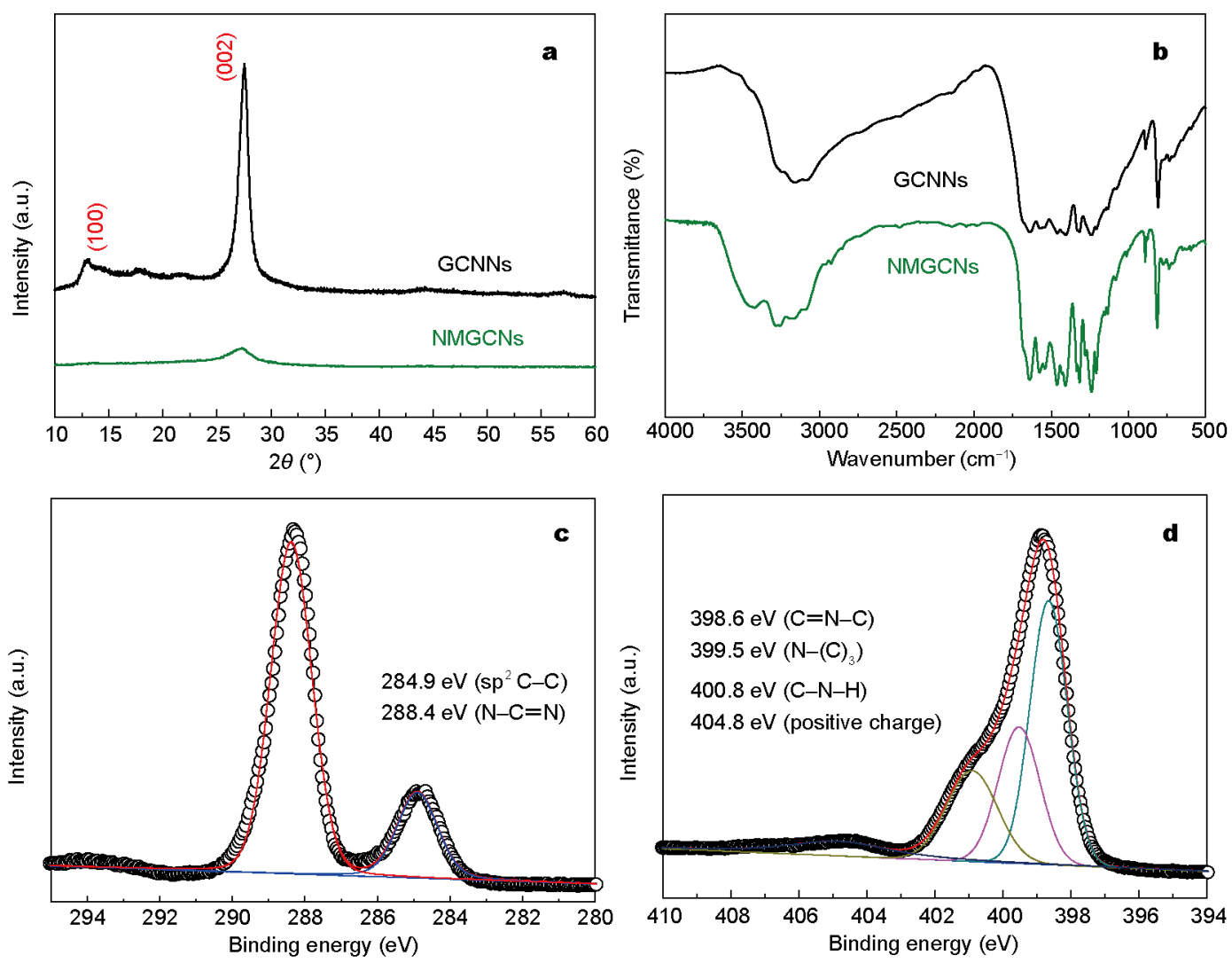

Figure 3 (a) XRD patterns and (b) FTIR spectra of GCNNs and NMGCNs. (c) C 1s and (d) N 1s XPS spectra of NMGCN. 
properties of the NMGCNs [16,39].

The as-prepared NMGCNs are well dispersed in water. A typical Tyndall effect observed in the aqueous solution with a green laser beam indicates a colloidal nature of the NMGCN dispersion (inset in Fig. 4a). The Zeta-potential of the NMGCNs dispersion in water is determined to be about $31 \mathrm{mV}$. The UV-vis absorption spectrum of the aqueous solution of NMGCNs shows two peaks centered at about 250 and $300 \mathrm{~nm}$, which are ascribed to the absorption from the $\pi-\pi^{*}$ of $\mathrm{C}=\mathrm{N}$ and $\mathrm{CN}$ heterocycles, respectively. This is also supported by mass spectrometry (MS) measurements. As shown in Fig. 4b, the MS of the NMGCN dispersion shows a dominant signal at $701 \mathrm{~mol} \mathrm{~g}^{-1}$, which is consistent with the relative molecular weight of the basic heptazine in GCN (inset in Fig. 4b). It must be mentioned that, as a result of the positive Zeta-potential $(+32.6$ $\mathrm{mV}$ ) and good stability of NMGCN dispersion, the absorption spectrum shows no obvious change after keeping the dispersion for six months at room temperature (Fig. S6). Moreover, the aqueous solution of NMGCNs emits bright blue fluorescence under UV light illumination (inset in Fig. 4a). As expected, a strong emission peak centered at about $432 \mathrm{~nm}$ was detected in the photoluminescence (PL) emission spectrum (Fig. 4a). The fluorescence intensity of the NMGCNs dispersion did not change significantly after continuous excitation with UV light for six hours and keeping it for six months, indicating that it has good structural stability and photostability (Fig. S7). Using quinine sulfate as the standard sample, the fluorescence quantum yield is determined to be about $32 \%$, which is much higher than that of ultrathin GCNNs (usually less than 20\%) [22], and similar to the values for the GCN quantum dots $[41,44]$. Moreover, the maximum PL emission position is independent of the wavelength of excitation light (Fig. S8), suggesting that the PL emission is ascribed to the intrinsic emission of the NMGCNs. To confirm this assumption, we estimated the bandgap of the NMGCNs by diffuse reflectance spectroscopy (DRS) measurements. As shown in Fig. 4c, the absorption edge of the NMGCN powder shows an obvious blue shift to $440 \mathrm{~nm}$ compared to that of the pristine GCNNs $(475 \mathrm{~nm})$. It was observed that the absorption edge of NMGCNs was close to the wavelength of the maximum PL emission. Accordingly, the energy band gaps of NMGCNs and GCNNs are respectively estimated to be 2.80 and 2.61 $\mathrm{eV}$ from the Tauc plots based on the Kubelka-Munk theory (inset in Fig. 4c). The wider bandgap of NMGCNs is ascri-
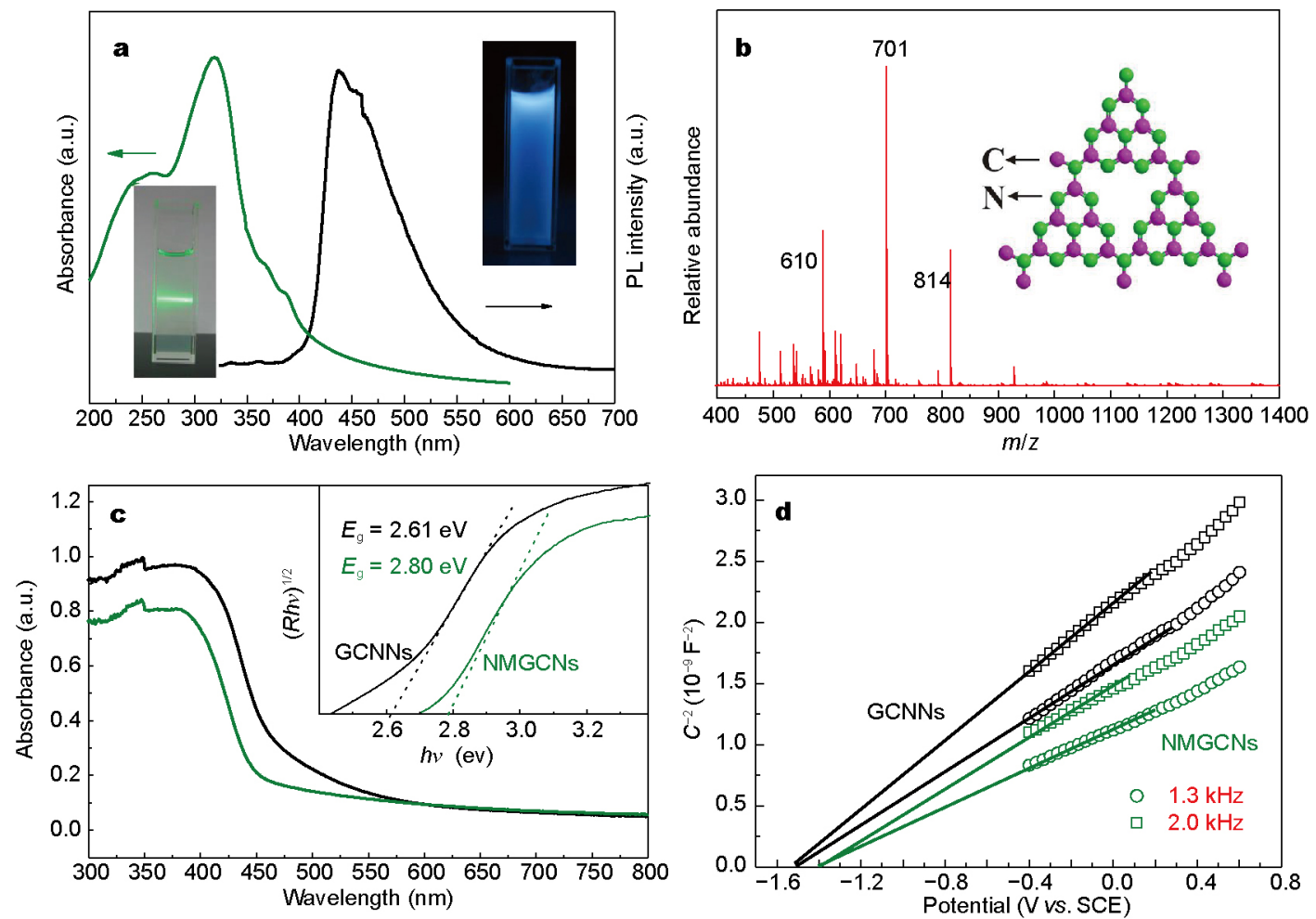

Figure 4 (a) UV-visible absorption spectra, the Tyndall effect, the fluorescence image under UV light illumination, and (b) mass spectrum of the NMGCNs dispersed in water. (c) UV-visible DRS, and (d) Mott-Schottky plots of GCNNs and NMGCNs. The inset in (b) is the heptazine structure of carbon nitrides. 
bed to the QCE arising from the small lateral size and monolayer structure [37]. In order to verify this deduction, we tested the Mott-Schottky plots by electrochemical method in detail. As compared in Fig. 4d, both NMGCNs and GCNNs exhibit positively sloping Mott-Schottky plots for two frequencies (1.3 and $2.0 \mathrm{kHz}$ ), suggesting that the NMGCN is still a kind of n-type semiconductor [29]. Furthermore, the derived flat-band potentials for GCNNs and NMGCNs are respectively about 1.50 and $1.39 \mathrm{~V}$ versus the saturated calomel electrode (SCE). Accordingly, the GCNN and NMGCN valence bands are about 1.11 and $1.41 \mathrm{~V}$ versus the SCE based on their bandgap values obtained by DRS (Fig. S9) [29]. It is found that both the valence and conduction bands of NMGCNs and GCNNs are significantly different, confirming that the QCE greatly influences the bandgap position. Remarkably, the NMGCNs are still thermodynamically suitable for achieving overall water splitting by photocatalysis since the valance and conduction bands straddle the water splitting redox potentials.

As mentioned above, the resulting NMGCNs have superior fluorescence properties, good dispersibility in water, and small lateral dimensions, enabling them to be used as a potential fluorescent marker for cell imaging. In this regard, a NMGCN aqueous solution was used to incubate A549 cells according to our previous method [45]. As shown in Fig. 5a, no fluorescence was observed in the bright-field image of the cells; however, they show bright blue fluorescence under UV light irradiation (Fig. 5b). A merging of the bright and dark images under UV light clearly shows that the blue fluorescence is located in the cytoplasm of the A549 cells (Fig. 5c), suggesting that the NMGCNs are able to enter into the cytoplasm of the cells. A standard MTT assay with A549 cells was carried out to evaluate the cytotoxicity of the NMGCNs [45]. As shown in Fig. 5d, more than $80 \%$ of the cells were still alive after incubation with the NMGCNs at concentrations ranging from 5 to $100 \mu \mathrm{g} \mathrm{mL}^{-1}$ for $48 \mathrm{~h}$, demonstrating the good biocompatibility of the NMGCNs. Good fluorescence properties and low cytotoxicity enable the NMGCNs to act as a metal-free bioimaging marker.

The NMGCN that has a small lateral size and a monolayer structure are also believed to be very useful for the application in photocatalysis since there are more exposed active sites and edges in contact with the reactant. The visible light driven $(\lambda \geq 400 \mathrm{~nm})$ decomposition of a Rhodamine $\mathrm{B}(\mathrm{RhB})$ aqueous solution was carried out to compare the photocatalytic activities of the NMGCNs and GCNNs in the same conditions. As shown in Fig. 6a, the blank test indicates that photo-degradation of RhB is hardly seen in the absence of any photocatalysts. With the NMGCNs, the absorbance of $\mathrm{RhB}$ decreased quickly under visible light
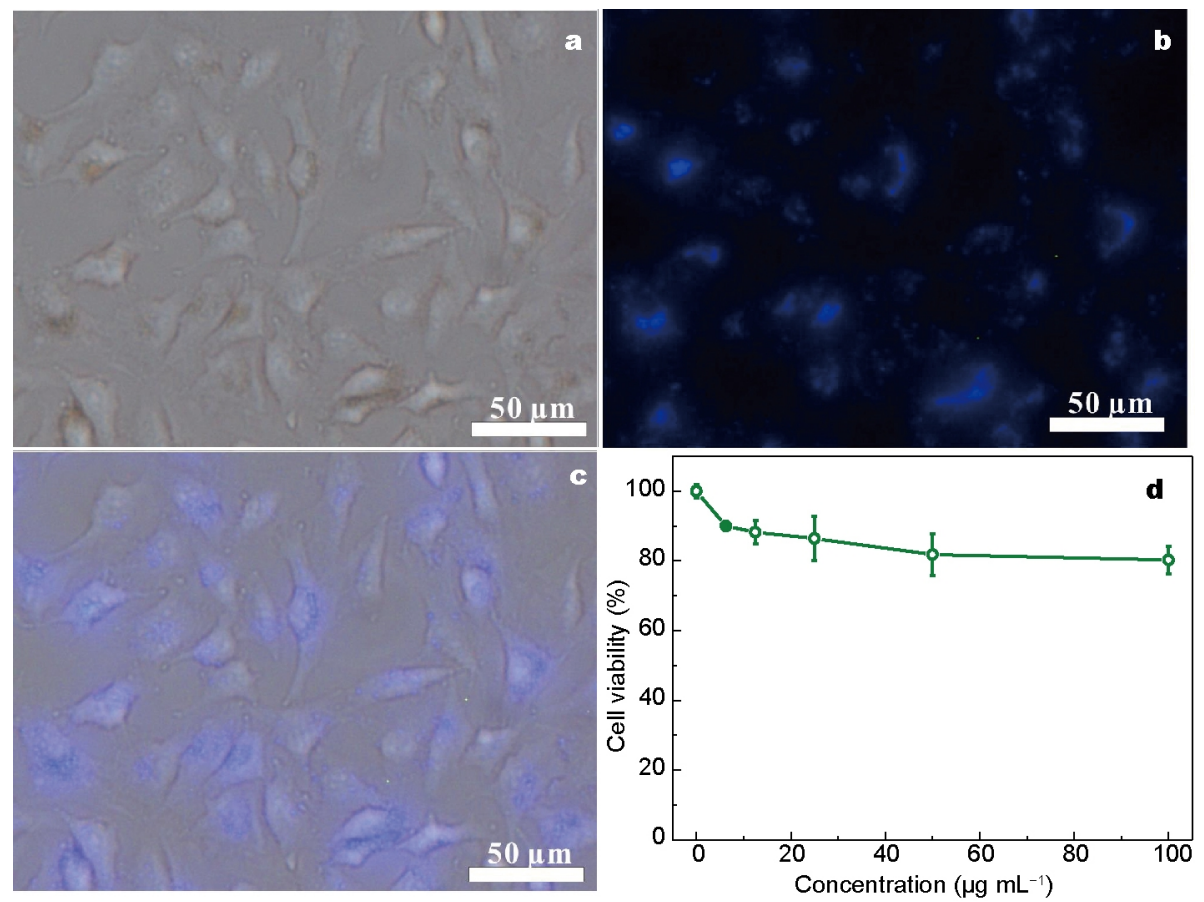

Figure 5 (a) Bright field image, (b) fluorescence image under UV light, and (c) the corresponding overlay images of A549 cells incubated with NMGCNs for $6 \mathrm{~h}$. (d) Viability of A549 cells incubated with different concentrations of NMGCNs for $48 \mathrm{~h}$. 

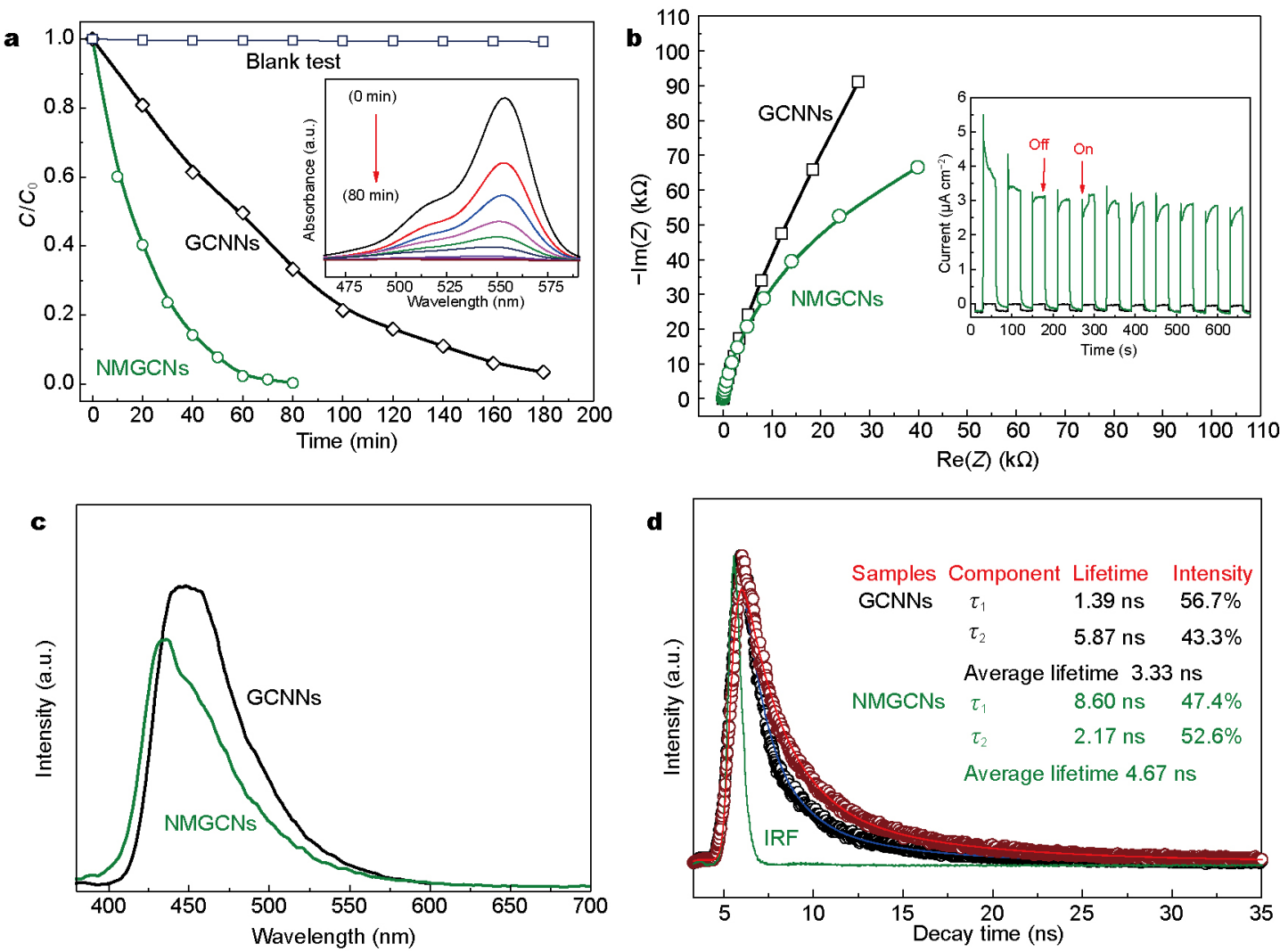

Figure 6 (a) Photocatalytic degradation curves, (b) EIS plots and photocurrent curves under visible light irradiation, (c) PL emission, and (d) the timeresolved fluorescence decay spectra of the NMGCN powder. The inset in (a) is the UV-Vis absorption spectra of RhB after degradation by NMGCNs for different time.

irradiation (inset in Fig. 6a). The time dependence of $\mathrm{RhB}$ photo-degradation reveals that almost $98 \%$ of the $\mathrm{RhB}$ aqueous solution was degraded over NMGCNs and GC$\mathrm{NNs}$ after 80 and 180 minutes, respectively. It is noted that, agreeing with previous report, the GCN quantum dots also show negligible photocatalytic activity under visible light, which is attributed to their ignorable photo-response under visible light [23]. The rate constants, quantitatively estimated by the kinetic principle of a pseudo first-order reaction are respectively 1.10 and $3.52 \mathrm{~h}^{-1}$ for the GCNNs and NMGCNs (Fig. S10). Generally, the photocatalytic activity of the NMGCNs is 3.3 times higher than that of the GCNNs.

We conducted photochemical measurements to study the photo-response behaviors of the NMGCNs and GCNNs. Compared with the GCNNs, the NMGCN shows a much smaller semicircle radius of the electrochemical impedance spectroscopy (EIS) plot, suggesting the smaller electronic resistance at the interface of the NMGCN/electrolyte. The time-related photocurrent was also measured to study the photo-response behaviors of the NMGCNs and GCNNs.
As compared in Fig. 6b, both GCNN and NMGCN electrodes show that the photocurrent rapidly increased when the light was turned on and then decreased quickly after turning it off. However, the maximum photocurrent of the NMGCNs $\left(\sim 3.2-4.0 \mu \mathrm{A} \mathrm{cm}^{-2}\right)$ is up to 20 times higher than that of GCNNs $\left(\sim 0.18-0.2 \mu \mathrm{A} \mathrm{cm}^{-2}\right)$, confirming the much better photo-response of the NMGCNs. Moreover, the process can be repeated ten times with no significant difference, suggesting the good stability of NMGCNs under visible light irradiation. The better photo-response of the NMGCNs is attributed to the improved separation efficiency of photoexcited charge carriers. As compared in Fig. 6c, the NMGCN powder shows a lower PL emission intensity than the GCNN case, suggesting the lower charge recombination rate in the NMGCNs [14,46,47]. A blue shift of the maximum emission peak was also observed in the PL spectrum of the NMGCN powder, which was in agreement with the above DRS results. Furthermore, time-resolved fluorescence spectroscopy reveals that both NMGCNs and GCNNs show a short $\left(\tau_{1}\right)$ and a long $\left(\tau_{2}\right)$ lifetime of charge carriers with different proportions (Fig. 
6d). Accordingly, the average PL lifetimes calculated by a double exponential fit are respectively 4.67 and 3.33 ns for the NMGCNs and GCNNs (Fig. 6d). The much increased lifetime of charge carriers in the NMGCNs is due to the smaller lateral size and monolayer structure, which result in reduced conjugation system with more exposed edges. Furthermore, the $\mathrm{N}_{2}$ adsorption measurements (Fig. S2) show that the SSA and pore volume of the NMGCN powder are about $220 \mathrm{~m}^{2} \mathrm{~g}^{-1}$ and $1.10 \mathrm{~cm}^{3} \mathrm{~g}^{-1}$, respectively. Both values are about two times of those for GCNNs $\left(102 \mathrm{~m}^{2} \mathrm{~g}^{-1}\right.$ and $\left.0.55 \mathrm{~cm}^{3} \mathrm{~g}^{-1}\right)$, confirming the presence of more photocatalytic active sites in the NMGCNs. This is further confirmed by the ferromagnetic measurements because theoretical and experimental studies suggest that the ultrathin GCNNs show intrinsic ferromagnetism at room temperature due to the presence of hydrogen dangling bonds $[28,48]$. As expected, the $M-H$ curves of NMGCNs and GCNNs show an obvious hysteresis loop with saturation magnetization (Fig. S11). However, the saturation magnetization $\left(M_{s}\right)$ value of the NMGCNs reaches $0.04 \mathrm{emu} \mathrm{g}^{-1}$, which is about five times larger than that for the GCNNs $\left(0.008 \mathrm{emu} \mathrm{g}^{-1}\right)$ and also much higher than previously reported values for the ultrathin GCNNs [28,48]. Notably, this is the highest $M_{s}$ value among the previous reports for GCN. Such a high $M_{s}$ of the NMGCNs is ascribed to the nanosized and monolayer structures, inducing abundant hydrogen dangling bonds and a higher spin density distribution and magnetic moment [28]. The intrinsic room temperature ferromagnetism with a high $M_{s}$ also makes the metal-free NMGCNs a promising nanomaterial for developing portable electronic and spintronic devices. In a word, the good photocatalytic performance and fluorescence of the NMGCNs are attributed to their highly improved photo-response, resulting from the unique monolayer and reduced-sized structures.

\section{CONCLUSIONS}

Nanosized monolayer carbon nitride nanosheets with a lateral size of about $55 \mathrm{~nm}$ and a thickness of $0.6 \mathrm{~nm}$ were prepared by using an easy yet efficient liquid-phase exfoliation method. As expected, the NMGCNs show excellent photo-responsive properties for both photocatalysis and fluorescence. As a result of the quantum size effect due to the nanosized and monolayer structure, a water dispersion of NMGCNs exhibits strong blue fluorescence with a high quantum yield of $32 \%$ under UV light, which is applicable as a good fluorescent marker for cell imaging. With a lateral size apparently larger than GCN quantum dots, NMGCNs also show remarkable photocatalytic performance under visible light irradiation. Therefore, with combined properties of sheet-like GCNNs and GCN quantum dots, NMGCNs show potential applications as sensors, energy storage and conversion devices, and electronic devices. This study also paves the way, towards some special applications, for the development of other mono-layered or ultrathin nanosheets with small lateral size, which fills the gap between high aspect ratio $2 \mathrm{D}$ materials and quantum dot-like counterparts.

Received 22 September 2016; accepted 31 October 2016; published online 13 December 2016

1 Zeng Q, Wang $\mathrm{H}, \mathrm{Fu} \mathrm{W}$, et al. Band engineering for novel twodimensional atomic layers. Small, 2015, 11: 1868-1884

2 Zhang H. Ultrathin two-dimensional nanomaterials. ACS Nano, 2015, 9: 9451-9469

3 Liu J, Cao H, Jiang B, et al. Newborn 2D materials for flexible energy conversion and storage. Sci China Mater, 2016, 59: 459-474

4 Liang L, Li K, Xiao C, et al. Vacancy associates-rich ultrathin nanosheets for high performance and flexible nonvolatile memory device. J Am Chem Soc, 2015, 137: 3102-3108

$5 \mathrm{Yu}$ XY, Hu H, Wang Y, et al. Ultrathin $\mathrm{MoS}_{2}$ nanosheets supported on $\mathrm{N}$-doped carbon nanoboxes with enhanced lithium storage and electrocatalytic properties. Angew Chem Int Ed, 2015, 54: 7395-7398

6 Tan C, Yu P, Hu Y, et al. High-yield exfoliation of ultrathin twodimensional ternary chalcogenide nanosheets for highly sensitive and selective fluorescence DNA sensors. J Am Chem Soc, 2015, 137: 10430-10436

7 Ma TY, Tang Y, Dai S, et al. Proton-functionalized two-dimensional graphitic carbon nitride nanosheet: an excellent metal-/label-free biosensing platform. Small, 2014, 10: 2382-2389

8 Zheng Y, Dou X, Li H, et al. Bisulfite induced chemiluminescence of g- $\mathrm{C}_{3} \mathrm{~N}_{4}$ nanosheets and enhanced by metal ions. Nanoscale, 2016, 8: 4933-4937

9 Xiang MH, Liu JW, Li N, et al. A fluorescent graphitic carbon nitride nanosheet biosensor for highly sensitive, label-free detection of alkaline phosphatase. Nanoscale, 2016, 8: 4727-4732

10 Wang S, Li K, Chen Y, et al. Biocompatible PEGylated $\mathrm{MoS}_{2}$ nanosheets: controllable bottom-up synthesis and highly efficient photothermal regression of tumor. Biomaterials, 2015, 39: 206-217

11 Ang H, Tan HT, Luo ZM, et al. Hydrophilic nitrogen and sulfur co-doped molybdenum carbide nanosheets for electrochemical hydrogen evolution. Small, 2015, 11: 6278-6284

12 Huang H, Feng X, Du C, et al. High-quality phosphorus-doped $\mathrm{MoS}_{2}$ ultrathin nanosheets with amenable ORR catalytic activity. Chem Commun, 2015, 51: 7903-7906

13 Lin Q, Li L, Liang S, et al. Efficient synthesis of monolayer carbon nitride 2D nanosheet with tunable concentration and enhanced visible-light photocatalytic activities. Appl Catal B-Environ, 2015, 163: $135-142$

14 Zhang M, Luo Z, Zhou M, et al. Photocatalytic water oxidation by layered Co/h-BCN hybrids. Sci China Mater, 2015, 58: 867-876

$15 \mathrm{Hu} \mathrm{C}$, Han Q, Zhao F, et al. Graphitic $\mathrm{C}_{3} \mathrm{~N}_{4}$-Pt nanohybrids supported on a graphene network for highly efficient methanol oxidation. Sci China Mater, 2015, 58: 21-27

16 Wang X, Maeda K, Thomas A, et al. A metal-free polymeric photocatalyst for hydrogen production from water under visible light. 
Nat Mater, 2009, 8: 76-80

17 Liu J, Liu Y, Liu N, et al. Metal-free efficient photocatalyst for stable visible water splitting via a two-electron pathway. Science, 2015, 347: 970-974

18 Zhang J, Chen Y, Wang X. Two-dimensional covalent carbon nitride nanosheets: synthesis, functionalization, and applications. Energ Environ Sci, 2015, 8: 3092-3108

19 Wang J, Shen Y, Li Y, et al. Crystallinity modulation of layered carbon nitride for enhanced photocatalytic activities. Chem Eur J, 2016, 22: 12449-12454

20 Liang Q, Li Z, Huang ZH, et al. Holey graphitic carbon nitride nanosheets with carbon vacancies for highly improved photocatalytic hydrogen production. Adv Funct Mater, 2015, 25: 6885-6892

21 Rong M, Cai Z, Xie L, et al. Study on the ultrahigh quantum yield of fluorescent $\mathrm{P}, \mathrm{O}-\mathrm{g}-\mathrm{C}_{3} \mathrm{~N}_{4}$ nanodots and its application in cell imaging. Chem Eur J, 2016, 22: 9387-9395

22 Zhang X, Xie X, Wang H, et al. Enhanced photoresponsive ultrathin graphitic-phase $\mathrm{C}_{3} \mathrm{~N}_{4}$ nanosheets for bioimaging. J Am Chem Soc, 2013, 135: 18-21

23 Wang W, Yu JC, Shen Z, et al. g- $\mathrm{C}_{3} \mathrm{~N}_{4}$ quantum dots: direct synthesis, upconversion properties and photocatalytic application. Chem Commun, 2014, 50: 10148-10150

24 Zhu K, Wang W, Meng A, et al. Mechanically exfoliated g- $\mathrm{C}_{3} \mathrm{~N}_{4}$ thin nanosheets by ball milling as high performance photocatalysts. RSC Adv, 2015, 5: 56239-56243

25 Han Q, Zhao F, Hu C, et al. Facile production of ultrathin graphitic carbon nitride nanoplatelets for efficient visible-light water splitting. Nano Res, 2015, 8: 1718-1728

26 Niu P, Zhang L, Liu G, et al. Graphene-like carbon nitride nanosheets for improved photocatalytic activities. Adv Funct Mater, 2012, 22: 4763-4770

27 Du X, Zou G, Wang Z, et al. A scalable chemical route to soluble acidified graphitic carbon nitride: an ideal precursor for isolated ultrathin $g-\mathrm{C}_{3} \mathrm{~N}_{4}$ nanosheets. Nanoscale, 2015, 7: 8701-8706

$28 \mathrm{Xu} \mathrm{K}, \mathrm{Li} \mathrm{X}$, Chen $\mathrm{P}$, et al. Hydrogen dangling bonds induce ferromagnetism in two-dimensional metal-free graphitic- $\mathrm{C}_{3} \mathrm{~N}_{4}$ nanosheets. Chem Sci, 2015, 6: 283-287

29 Yang S, Gong Y, Zhang J, et al. Exfoliated graphitic carbon nitride nanosheets as efficient catalysts for hydrogen evolution under visible light. Adv Mater, 2013, 25: 2452-2456

30 Lu Q, Deng J, Hou Y, et al. One-step electrochemical synthesis of ultrathin graphitic carbon nitride nanosheets and their application to the detection of uric acid. Chem Commun, 2015, 51: 12251-12253

31 Tian J, Liu Q, Asiri AM, et al. Ultrathin graphitic carbon nitride nanosheets: a novel peroxidase mimetic, Fe doping-mediated catalytic performance enhancement and application to rapid, highly sensitive optical detection of glucose. Nanoscale, 2013, 5: 11604-11609

32 Tian J, Liu Q, Asiri AM, et al. Ultrathin graphitic $\mathrm{C}_{3} \mathrm{~N}_{4}$ nanosheets/graphene composites: efficient organic electrocatalyst for oxygen evolution reaction. ChemSusChem, 2014, 7: 2125-2130

33 Tong J, Zhang L, Li F, et al. An efficient top-down approach for the fabrication of large-aspect-ratio $\mathrm{g}-\mathrm{C}_{3} \mathrm{~N}_{4}$ nanosheets with enhanced photocatalytic activities. Phys Chem Chem Phys, 2015, 17: 23532-23537

34 Zhao $\mathrm{H}$, Yu H, Quan X, et al. Atomic single layer graphitic- $\mathrm{C}_{3} \mathrm{~N}_{4}$ : fabrication and its high photocatalytic performance under visible light irradiation. RSC Adv, 2014, 4: 624-628

35 Sun Z, Xie H, Tang S, et al. Ultrasmall black phosphorus quantum dots: synthesis and use as photothermal agents. Angew Chem Int Ed, 2015, 54: 11526-11530

36 Liang Q, Ye L, Xu Q, et al. Graphitic carbon nitride nanosheetassisted preparation of $\mathrm{N}$-enriched mesoporous carbon nanofibers with improved capacitive performance. Carbon, 2015, 94: 342-348

37 Yuan B, Chu Z, Li G, et al. Water-soluble ribbon-like graphitic carbon nitride $\left(\mathrm{g}-\mathrm{C}_{3} \mathrm{~N}_{4}\right)$ : green synthesis, self-assembly and unique optical properties. J Mater Chem C, 2014, 2: 8212-8215

38 Liang Q, Huang ZH, Kang F, et al. Facile synthesis of crystalline polymeric carbon nitrides with an enhanced photocatalytic performance under visible light. ChemCatChem, 2015, 7: 2897-2902

39 Cao S, Low J, Yu J, et al. Polymeric photocatalysts based on graphitic carbon nitride. Adv Mater, 2015, 27: 2150-2176

40 Rong M, Song X, Zhao T, et al. Synthesis of highly fluorescent $\mathrm{P}, \mathrm{O}-\mathrm{g}-\mathrm{C}_{3} \mathrm{~N}_{4}$ nanodots for the label-free detection of $\mathrm{Cu}^{2+}$ and acetylcholinesterase activity. J Mater Chem C, 2015, 3: 10916-10924

41 Lu YC, Chen J, Wang AJ, et al. Facile synthesis of oxygen and sulfur co-doped graphitic carbon nitride fluorescent quantum dots and their application for mercury(II) detection and bioimaging. J Mater Chem C, 2015, 3: 73-78

42 Liang Q, Li Z, Yu X, et al. Macroscopic 3D porous graphitic carbon nitride monolith for enhanced photocatalytic hydrogen evolution. Adv Mater, 2015, 27: 4634-4639

43 Zhang J, Zhang M, Yang C, et al. Nanospherical carbon nitride frameworks with sharp edges accelerating charge collection and separation at a soft photocatalytic interface. Adv Mater, 2014, 26: 4121-4126

44 Zhang S, Li J, Zeng M, et al. Polymer nanodots of graphitic carbon nitride as effective fluorescent probes for the detection of $\mathrm{Fe}^{3+}$ and $\mathrm{Cu}^{2+}$ ions. Nanoscale, 2014, 6: 4157-4162

45 Liang Q, Ma W, Shi Y, et al. Easy synthesis of highly fluorescent carbon quantum dots from gelatin and their luminescent properties and applications. Carbon, 2013, 60: 421-428

46 Li X, Wen J, Low J, et al. Design and fabrication of semiconductor photocatalyst for photocatalytic reduction of $\mathrm{CO}_{2}$ to solar fuel. Sci China Mater, 2014, 57: 70-100

47 Low J, Cheng B, Yu J, et al. Carbon-based two-dimensional layered materials for photocatalytic $\mathrm{CO}_{2}$ reduction to solar fuels. Energ Storage Mater, 2016, 3: 24-35

48 Gao D, Xu Q, Zhang J, et al. Defect-related ferromagnetism in ultrathin metal-free g- $\mathrm{C}_{3} \mathrm{~N}_{4}$ nanosheets. Nanoscale, 2014, 6: 2577-2581

Acknowledgments This work was supported by the National Basic Research Program of China (2014CB932400), the National Natural Science Foundation of China (51525204 and 51302274), Shenzhen Basic Research Project (ZDSYS20140509172959981), and the Key Laboratory of Advanced Materials of Ministry of Education (2016AML02).

Author contributions All authors contributed to the discussion and preparation of the manuscript. The final version of the manuscript was approved by all authors.

Conflict of interest The authors declare that they have no conflict of interest.

Supplementary information Experimental details and supporting data are available in the online version of the paper. 

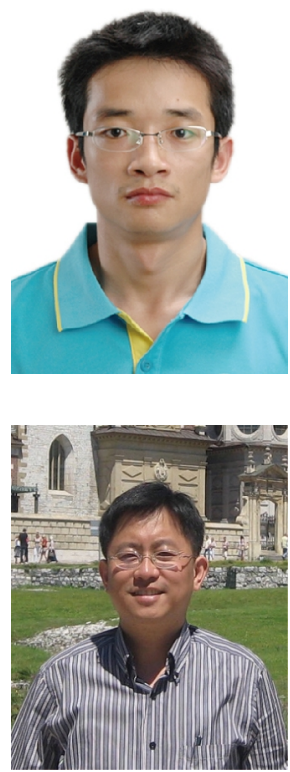

Qinghua Liang received his Bachelor's degree from Southwest University in 2009 and Master's degree from Technical Institute of Physics and Chemistry of Chinese Academy of Sciences in 2012. He obtained his PhD from Tsinghua University under the supervision of Prof. Quan-Hong Yang. His research interest focuses on the synthesis and application of carbon-based and carbon-derived materials for energy storage and environmental protection.

Quan-Hong Yang was born in 1972 and joined Tianjin University as a full professor of nanomaterials in 2006. He is now also leading a graphene lab as a co-PI at Tsinghua-Berkeley Shenzhen Institute (TBSI). His research is totally related to novel carbon materials, from porous carbons, tubular carbons to sheet-like graphenes with their applications in energy storage and environmental protection. See http://nanoyang.tju.edu.cn for more details.

\section{具有显著增强光响应的小尺寸单层石墨相氮化碳纳米片用于细胞成像及光催化}

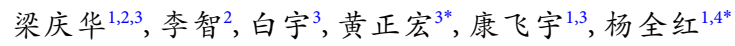

摘要 不含金属的二维石墨相氮化碳纳米片由于具有优异的催化、光学及电学性能而受到研究者的广泛关注. 然而制备纳米级尺寸的单 层石墨相氮化碳纳米片仍然存在挑战. 本文采用一种高效超声方法制备了横向尺寸约为 $55 \mathrm{~nm}$, 厚度约为 $0.6 \mathrm{~nm}$ 的单层石墨相氮化碳纳米 片(NMGCNs). 由于其纳米级尺寸及单层片状结构, NMGCNs 表现出与大尺寸纳米片和量子点显著不同的光响应特性. NMGCNs的水分散 溶液具有良好的稳定性能和优异的荧光性能, 荧光量子产率可达 $32 \%$, 所以可用于细胞荧光成像. 此外, NMGCNs表现出比多层石墨相氮化 碳纳米片更优异的可见光催化性能. 独特的小尺寸及单层超薄结构使得NMGCNs在传感器和光电子等领域都具有潜在应用前景. 